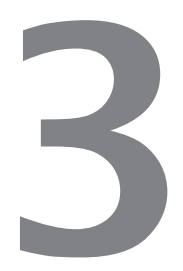

\title{
Rigidez geométrica e aspectos da estabilidade estrutural
}

Uma vez que o efeito da força axial de compressão é o de reduzir a rigidez dos membros da estrutura, a abordagem dos aspectos que envolvem o conceito de rigidez geométrica está relacionada, ao mesmo tempo, à análise da estabilidade elástica dos sistemas estruturais. A rigidez geométrica é uma função do esforço normal presente nos elementos estruturais. Expressões que deixam clara essa relação são apresentadas mais adiante nesta seção e no capítulo destinado à proposta de cálculo da frequência com a consideração da não-linearidade geométrica, apresentado no item 4. Excluindo-se a possibilidade de uma apresentação pormenorizada da teoria das vibrações, fez-se a introdução da não- 
-linearidade geométrica na dinâmica estrutural no que concerne aos objetivos deste estudo.

As considerações sobre estabilidade são preponderantes na análise de muitas estruturas da engenharia porque essas são normalmente projetadas para suportar cargas com pequenas e limitadas deformações. Souza Lima e Venâncio Filho (1982) afirmam que as considerações da não-linearidade geométrica interessam a dois tipos de problema, aos que se prendem ao cálculo dos esforços de segunda ordem em estruturas de rigidez reduzida e aos que se ligam diretamente aos fenômenos de perda de instabilidade do equilíbrio por flambagem ou aparecimento de ponto limite na configuração de equilíbrio.

Importantes nomes da Física e da Matemática se sentiram atraídos por investigar o tema da estabilidade. Os primeiros estudos estão ligados a Aristóteles e Arquimedes. Nomes como os de Euler, Torricelli, Baldi, Timoshenko, Lagrange, Lamarle, Baushinger, Considère, Tetmajer, Liapunov, Hellinger, Bryan e Nicolai também aparecem como cientistas que aportaram significativas contribuições, APUD Mazzilli (1979). Mais recentemente, Ratzersdorfer (1954) apresentou abrangente estudo sobre o assunto.

A estabilidade é entendida como a tendência de um sistema estrutural em persistir em determinado estado, quando estiver sob a influência de pequenas perturbações externas agindo no sentido de encorajá-lo a abandonar tal estado. Uma configuração que não é estável é dita instável. A perda de estabilidade depende das propriedades do material, da configuração estrutural e das condições do carregamento. A instabilidade que ocorre sob ação de carga compressiva é comumente conhecida como flambagem.

A falência por flambagem é potencialmente perigosa e pode iniciar a ruína de muitos tipos de estruturas da engenharia, podendo dar-se em relação a toda a estrutura ou localmente em relação a componentes individuais, e, mesmo nesses casos, induzir a estrutura ao colapso. Para Gambir (2004) é importante destacar que a carga para a qual ocorre a flambagem depende mais da rigidez da estrutura do que da resistência dos materiais. Fusco (1981) acrescenta que para os materiais estruturais como o concreto e o aço, o estado limite de flambagem é um estado limite último. 
A análise da estabilidade consiste em se determinar o modo da perda da estabilidade e a correspondente carga crítica. A estrutura permanece em repouso antes e depois da flambagem, exceto nos casos nos quais a flambagem ocorre devido à transição do estado de repouso para o movimento, chamada instabilidade cinética ou dinâmica.

As primeiras postulações analíticas para o entendimento do fenômeno da flambagem em barras comprimidas axialmente devem-se a Euler, APUD Timoshenko (1936), que considerou o pilar como uma barra prismática de eixo reto, submetida a uma força axial aplicada no centro de gravidade da seção. Dessa maneira, Euler resolveu o problema das barras comprimidas tomando como referência um pilar ideal, i.e., uma barra sem imperfeições geométricas nas suas condições iniciais, comprimida por uma carga aplicada no seu eixo e cujo material fosse perfeitamente elástico-linear.

As formulações analíticas da estática desenvolvidas por Euler representam a solução exata para o problema da estabilidade e, por conseguinte, têm servido de aferição às formulações desenvolvidas por outros métodos. Elas abordam o problema levando em conta duas hipóteses. A primeira restringi-se aos pequenos deslocamentos, enquanto que a segunda admite que o equilíbrio se dê na configuração deformada, a qual pode diferenciar-se consideravelmente da configuração de referência. Nesse caso, a carga crítica é aquela capaz de manter tal configuração. Os estudos feitos no presente trabalho limitam-se ao primeiro caso.

Considere-se o caso de uma barra prismática esbelta engastada na base e carregada axialmente na extremidade superior, como indica a Figura 3.1. Para valores de $P$ abaixo da carga crítica a configuração reta de equilíbrio permanece (Figura 3.1a), porém quando é atingida a carga crítica de flambagem o equilíbrio torna-se instável e uma pequena força lateral poderá produzir um deslocamento que não desaparecerá com a causa que o produziu. A carga crítica é, então, definida como a carga axial suficiente para manter a barra estável com a forma levemente fletida (Figura 3.1b).

Nesse caso, a carga crítica é calculada pelo emprego da equação aproximada da linha elástica $\frac{\mathrm{M}}{\mathrm{EI}}=\frac{\mathrm{d}^{2} \mathrm{y}}{\mathrm{dx}^{2}}$, na qual E é o módulo de 
elasticidade do material, I é a menor inércia, e M é o momento fletor em qualquer seção transversal mn dado por $\mathrm{P}(\delta-\mathrm{y})$. O desenvolvimento da expressão anterior conduz a uma equação diferencial a coeficientes constantes.

Resolvendo a equação diferencial da linha elástica resultante da introdução de M, com a observância das condições de contorno do problema, chega-se à conhecida expressão da carga crítica de Euler (Timoshenko,1936).

$$
\mathrm{P}_{\mathrm{cr}}=\frac{\pi^{2} \mathrm{EI}}{4 \mathrm{~L}^{2}}
$$

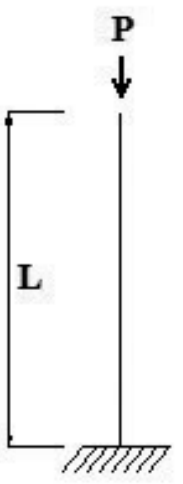

(a)

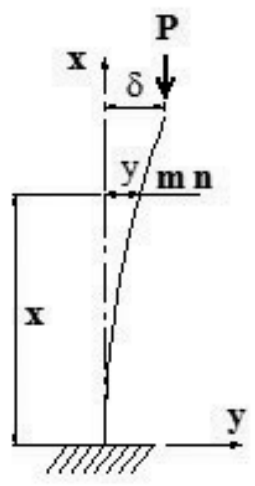

(b)

Figura 3.1 - Carga crítica de Euler - Timoshenko (1936).

A análise da estabilidade pelo método estático com a consideração do peso próprio dos elementos estruturais foi discutida inicialmente por Euler, que não teve sucesso na obtenção de uma solução satisfatória, sendo resolvido definitivamente por Greenhill em 1881 (TIMOSHENKO, 1936).

Para introduzir a ação de uma carga axial distribuída tome-se a barra da Figura 3.2 engastada na extremidade inferior e livre na superior. A obtenção da equação diferencial da curvatura para o caso de 
uma força compressiva uniformemente distribuída não é tão simples quando comparada à equação diferencial a coeficientes constantes. Nesse caso, a solução normalmente exige a aplicação de séries infinitas ou baseia-se em métodos aproximados, como por exemplo, o método da energia.

Se a barra da Figura 3.2, tida no início como verticalmente reta, estiver sob a ação de uma força uniforme axialmente distribuída, a equação diferencial da curvatura será dada por:

$$
E I \frac{d^{2} y}{d x^{2}}=\int_{x}^{L} q(\eta-y) d \xi
$$

onde a integral do lado direito da equação representa o momento fletor em uma seção qualquer devido à carga uniformemente distribuída q.

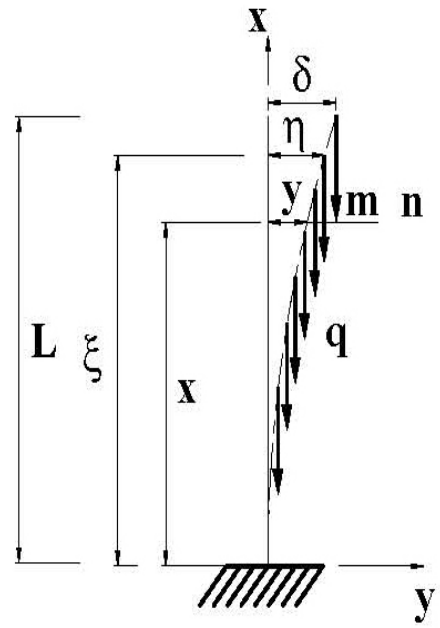

Figura 3.2 - Consideração do peso próprio da barra Timoshenko (1936).

Nesse caso, para a barra da Figura 3.2, pode ser tomada, como uma primeira aproximação para o cálculo dos deslocamentos laterais, a seguinte expressão: 


$$
\mathrm{y}=\delta\left(1-\cos \frac{\pi \mathrm{x}}{2 \mathrm{~L}}\right)
$$

que representa o valor exato para o caso de uma força concentrada de compressão aplicada na extremidade da barra e que conduz à expressão (3.1).

Embora a solução do problema de uma barra sujeita a seu peso próprio seja mais complicada, a equação (3.3) satisfaz às condições de contorno, sendo, por essa razão, apropriada para a maioria das situações práticas da engenharia.

Aplicando o Princípio da Conservação da Energia chega-se à expressão (3.4), que representa uma primeira aproximação do valor da carga crítica devido ao peso próprio

$$
(q \mathrm{~L})_{\mathrm{cr}}=\frac{7,89 \mathrm{EI}}{\mathrm{L}^{2}}
$$

A expressão (3.4) representa uma diferença de 0,77\%, quando comparada à solução obtida pelo método de séries infinitas, equação (3.5).

$$
(q L)_{c r}=\frac{7,837 E I}{L^{2}}=\frac{\pi^{2} \mathrm{EI}}{(1,122 \mathrm{~L})^{2}}
$$

De fato, a carga axial distribuída reduz o valor da carga crítica que pode ser aplicada na extremidade da barra. Fazendo-se 


$$
\mathrm{P}_{\mathrm{cr}}=\frac{\pi^{2} \mathrm{EI}}{4 \mathrm{~L}^{2}}=\frac{\mathrm{mEI}}{\mathrm{L}^{2}}
$$

verifica-se que o fator $m$, menor que $\pi^{2} / 4$, gradualmente diminui com o aumento de qL, se aproxima de zero quando qL se aproxima do valor dado em (3.5). Usando a notação

$$
n=\frac{q L}{\frac{\pi^{2} E I}{4 L^{2}}}
$$

pode-se calcular m para os vários valores de n. Timoshenko (1936) apresenta na Tabela 3.1 os valores de m calculados pela expressão (3.5) .

O interesse em mostrar o desenvolvimento apresentado por Timoshenko (1936) para o caso de uma barra sujeita a seu peso próprio está em verificar que, pela Tabela 3.1, é uma aproximação satisfatória assumir que o efeito do peso próprio, na magnitude da carga crítica, corresponde a 0,315qL aplicado na extremidade da barra. Isso implica em dizer que a carga crítica de Euler (3.1) será reduzida desse valor quando for levado em conta o peso próprio na determinação da carga crítica de flambagem.

\section{Tabela 3.1 - Relação entre o peso próprio e carga de Euler - Timoshenko (1936).}

\begin{tabular}{|c|c|c|c|c|c|c|c|c|c|c|c|}
\hline $\mathrm{n}$ & 0 & 0,25 & 0,5 & 0,75 & 1,0 & 2,0 & 3,0 & 3,177 & 4 & 5 & 10 \\
\hline $\mathrm{m}$ & 2,47 & 2,28 & 2,08 & 1,91 & 1,72 & 0,96 & 0,15 & 0 & $-0,69$ & $-1,56$ & $-6,95$ \\
\hline
\end{tabular}

Quando se analisa, sob a hipótese de pequenos deslocamentos, a influência do peso próprio na carga crítica de flambagem, verifica-se que quando o valor qL supera o valor dado pela expressão (3.5), $\mathrm{P}_{\mathrm{Cr}}$ de (3.1), torna-se negativo, o que implica em dizer que uma força 
axial de tração deve ser aplicada para evitar a flambagem da barra. Na hipótese de comportamento linear, o gráfico da Figura 3.3 permite acompanhar a variação da carga crítica de Euler, com e sem o peso próprio, em função do comprimento da barra.

Carga critica de Euler sob comportamento linear

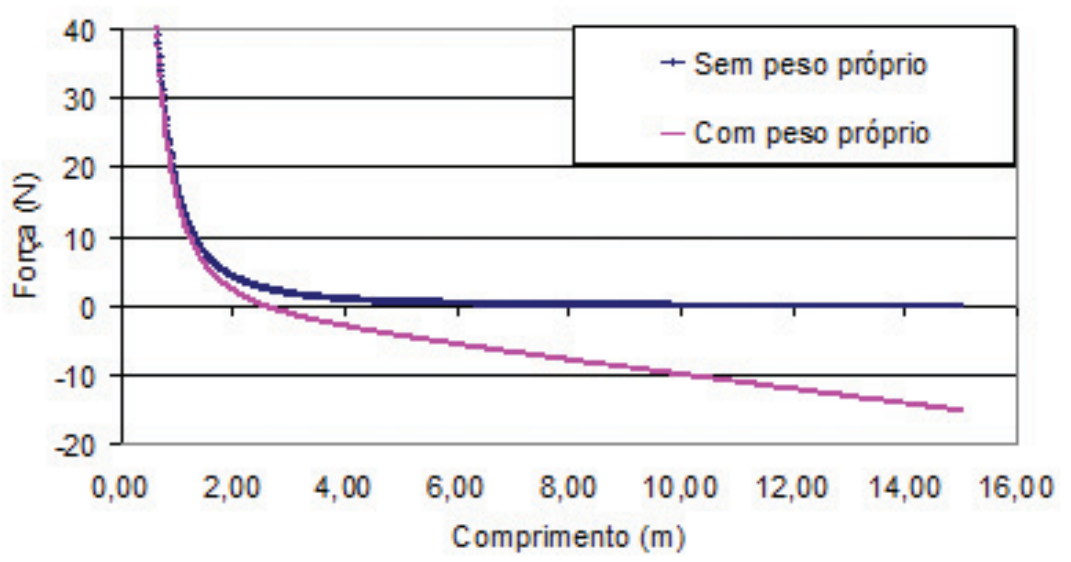

Figura 3.3 - Carga crítica levando com o peso próprio e carga crítica de Euler.

Nessa simulação foi adotado o mesmo módulo de elasticidade e a geometria da barra usada nos ensaios de laboratório, que serão apresentados na seção 5. Observa-se que devido à influência do peso próprio, a barra atinge a situação crítica da carga de compressão no comprimento de 2,56 m.

$\mathrm{Na}$ abordagem da estabilidade pelo Método dos Deslocamentos em formulação matricial Gambir (2004) se refere à influência da força axial como aquela que diminui a rigidez dos membros estruturais. Com isso, o efeito das cargas aplicadas sobre a estrutura aumenta, aumentando também as forças nos elementos, e a capacidade de resistência da estrutura a qualquer perturbação de natureza aleatória diminui. Para a carga de flambagem, a estrutura não oferece resistência a nenhuma perturbação que ocorra sobre ela e, em ocorrendo tal perturbação, os deslocamentos na configuração seguem aumentando sem a necessidade do acréscimo de cargas adicionais. Isso sugere que, quando se atinja esse instante, os deslocamentos da estrutura, para a carga crítica, crescerão indefinidamente, significando que, por outro lado, a rigidez da estrutura tornou-se nula. 
O critério da estabilidade elástica pelo Método dos Deslocamentos em formulação matricial pode ser entendida pela exposição que se segue.

A relação entre as forças externas aplicadas e os deslocamentos nodais é dada por

$$
\{F\}=[K]\{Q\},
$$

onde

$\{\mathrm{F}\}$ é o vetor de cargas nodais

$[\mathrm{K}]$ é a matriz de rigidez da estrutura

$\{\mathrm{Q}\}$ é o vetor deslocamento

Os deslocamentos nodais tornam-se então

$$
\{\mathrm{Q}\}=\left[\frac{\operatorname{Adj}[\mathrm{K}]}{|\mathrm{K}|}\right]\{\mathrm{F}\},
$$

onde

$\operatorname{Adj}[\mathrm{K}]$ é a matriz adjunta da matriz de rigidez, e

$|\mathrm{K}|$ é o determinante da matriz de rigidez.

Pela equação (3.8) para que os deslocamentos cresçam indefinidamente é preciso que o determinante da matriz de rigidez tenda a zero. Portanto, o critério de instabilidade é a condição de

$$
|\mathrm{K}|=0 \text {, }
$$

o que significa que matriz de rigidez seja singular. 
A equação característica resultante de (3.9) deve apresentar mais de uma solução para o critério de instabilidade, mas a menor deles, logicamente, é o que corresponderá à carga crítica de estabilidade. A matriz de rigidez deve ser vista como composta por dois termos, sendo um deles o correspondente à parcela da rigidez geométrica. Dessa forma, a análise da carga crítica de flambagem envolve a solução de um problema de autovalores na forma de

$$
\left(\left[\mathrm{K}_{0}\right]-\lambda_{\mathrm{r}}\left[\mathrm{K}_{\mathrm{g}}(\mathrm{r})\right]\right) \psi=0
$$

onde os termos entre colchetes representam as parcelas da matriz de rigidez, $\mathrm{l}_{\mathrm{r}}$ são os autovalores associados aos autovetores y correspondentes à força $r$. Os autovalores devem ser entendidos como um fator de multiplicação da carga $r$ para que se atinja a flambagem. Com isso, a interpretação dada aos autovalores é a de um fator de segurança em relação à força analisada, podendo ser maior ou menor que a unidade e até assumir valores negativos. Os autovalores mais altos correspondem às diversas condições de restrições externas e, portanto, inválidas caso essas restrições não existam.

Com a Equação (3.10) fica claro que a matriz de rigidez para estruturas com elementos sujeitos às cargas axiais é diferente da matriz de rigidez elástica, e que a matriz apresentada em (3.7) deve incluir seus efeitos, quer seja para minorar, como no caso da força de compressão, ou majorar, como no caso da força de tração, a rigidez dos sistemas estruturais.

Já na análise dinâmica da instabilidade, a carga crítica é obtida a partir da equação do movimento por meio de uma solução não-trivial por autovalores. Quando ocorre a perda da estabilidade a frequência de vibração natural da estrutura tende a zero. A investigação dinâmica da instabilidade é mais abrangente do que outros métodos de análise nos quais as forças de inércia são desprezadas, como na análise estática. Assim, uma vez que o método dinâmico leva em conta as forças de inércia na formulação da análise da estabilidade das estruturas, torna-se importante a maneira como se dá a distribuição de massa e a rigidez dos sistemas elásticos (GAMBIR, 2004). 
Clough (1993) ratifica a ideia de que a força axial ou qualquer força que tenda a produzir flambagem pode interferir significativamente na rigidez da estrutura. Segundo ele, a componente de uma força agindo paralelamente ao eixo originalmente vertical de elementos estruturais pode produzir efeitos adicionais nos deslocamentos nodais, ou nos extremos, desses elementos, e, portanto, a matriz de rigidez da estrutura deve ser composta por duas parcelas, uma elástica e outra geométrica. A partir daí, os modos e frequências de vibração de uma estrutura que esteja sujeita a um carregamento axial devem ser calculados exatamente da mesma forma como para sistemas que não levam em conta esses efeitos.

Levy (1994) apresenta um teorema que representa, em termos da teoria das estruturas, a importância da não-linearidade geométrica: "Na presença de tensões iniciais, não-linearidades geométricas são da mesma ordem de grandeza dos efeitos elásticos na estrutura".

Esse teorema implica em afirmar que, na maioria dos casos, e para todos os casos de análise incremental, os efeitos decorrentes das não-linearidades geométricas devem ser levados em consideração; e que problemas envolvendo flambagem de colunas, cabos e estruturas esbeltas exigem a inclusão de hipóteses que levem em conta a não-linearidade geométrica do comportamento estrutural.

Na consideração dessas hipóteses na análise da estabilidade dos sistemas estruturais, desde que a análise não dependa das condições da configuração de inicial ou de referência, Levy (2004) constrói uma formulação utilizando o conceito de operadores geométricos. Nesse caso, a equação de equilíbrio para um sistema deve ser escrita como

$$
\mathrm{C}^{\mathrm{T}} \mathrm{F}=\mathrm{P}
$$

onde $\mathrm{P}$ representa as cargas aplicadas, F representa as forças ou tensões internas, e C representa um operador que descreve o equilíbrio do sistema.

Essa equação pode também ser escrita através de equações matriciais de equilíbrio relativas aos nós de um sistema discreto. Se o sistema é perturbado com uma carga dP a sua resposta é dada por 


$$
d C^{\mathrm{T}} \mathrm{F}+\mathrm{C}^{\mathrm{T}} \mathrm{dF}=\mathrm{dP}
$$

O primeiro termo da equação (3.12) descreve a não-linearidade geométrica e o segundo termo diz respeito à teoria linear. Para um sistema discreto pode-se converter com relativa facilidade a equação anterior à forma conhecida do método dos deslocamentos

$$
\left(\mathrm{K}_{\mathrm{E}}+\mathrm{K}_{\mathrm{G}}\right) \delta=\mathrm{dP}
$$

onde $\mathrm{K}_{\mathrm{E}}$ e $\mathrm{K}_{\mathrm{G}}$ são as matrizes de rigidez elástica e geométrica e $\delta$ representa o deslocamento do sistema. Com essa aproximação análises não-lineares tornam-se simples aplicações do Método de Newton, que tem a vantagem de oferecer uma rápida convergência e as soluções das equações não-lineares podem ser obtidas com certa facilidade, continua Levy (1995).

Pauletti (2003) quando estuda estruturas retesadas ratifica a concepção de que o comportamento das estruturas são em maior ou menor grau não-linear. A importância da não-linearidade depende do tipo de estrutura e da fase de análise.

Para ampliar o entendimento do comportamento não-linear e a influência da rigidez geométrica é válido apresentar o trabalho que Pauletti (2003) desenvolve para uma corda (Figura 3.4), que nada mais é que um elemento de barra, estendido entre dois pontos fixos que suporta exclusivamente a força normal de tração $\mathrm{F}_{0}$ dada por

$$
\mathrm{F}_{0}=\frac{\mathrm{EA}}{\mathrm{L}_{\mathrm{r}}}\left(\mathrm{L}-\mathrm{L}_{\mathrm{r}}\right)=\mathrm{k}\left(\mathrm{L}-\mathrm{L}_{\mathrm{r}}\right)
$$

onde $\mathrm{E}$ é o módulo de elasticidade do material, A é área de sua seção transversal, L é a distância entre os pontos fixação, igual ao comprimento deformado, na configuração retilínea e $\mathrm{L}_{\mathrm{r}}$ é o comprimento indeformado do cabo. A constante $\mathrm{k}=\frac{\mathrm{EA}}{\mathrm{L}_{\mathrm{r}}}$ é chamada constante de mola. 


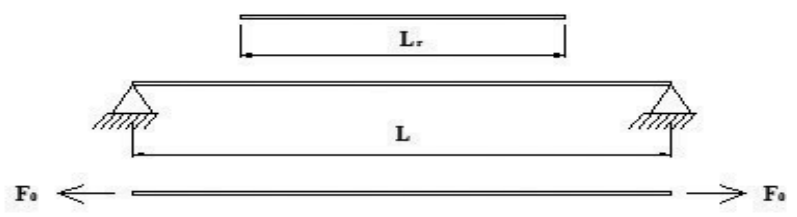

Figura 3.4 - Corda de comprimento indeformado Lr, tracionada entre dois apoios fixos distantes $L>L r-$ Pauletti (2003).

Como a rigidez transversal da corda em torno da sua configuração retilínea não depende de deformações adicionais impostas, mas sim de sua relutância em alterar a geometria, diz-se que essa é exclusivamente geométrica.

Desprezando-se o peso próprio e demais cargas externas, a configuração retilínea inicial (Figura 3.5) é uma configuração de equilíbrio sob a ação da força de tração. Se, porém, como é usual na análise linear de estruturas, tentar-se expressar a deflexão da corda, decorrente da imposição de um carregamento transversal, em termos da configuração inicial, retilínea, chega-se obviamente a uma indeterminação.

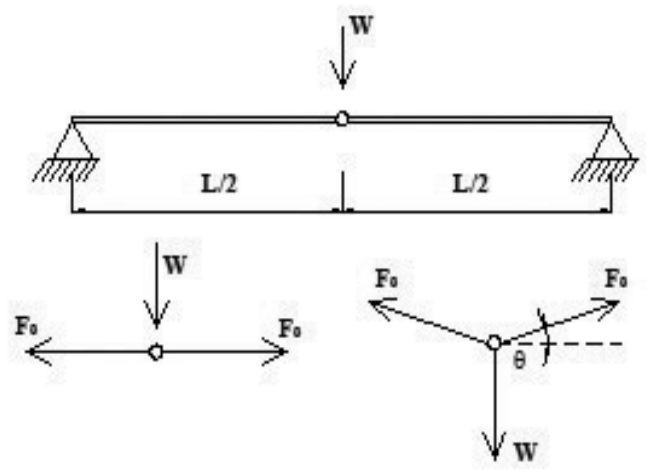

Figura 3.5 - Para a corda carregada transversalmente, o equilíbrio só é possível na configuração deformada - Pauletti (2003). 
Para entender o conceito de rigidez geométrica e elaborar a formulação da hipótese não-linear é preciso definir a força desbalanceada $\mathrm{g}(\mathrm{u})$ como a diferença entre a resultante das forças internas restauradoras e um carregamento externo $\mathrm{W}$, de modo que se tem $g(u)=f(u)-W$.

Em muitas ocasiões é interessante decompor a força desbalanceada em um esforço interno $\mathrm{F}=\mathrm{F}(\mathrm{u})$, e um operador geométrico $\mathrm{C}=\mathrm{C}(\mathrm{u})$, aplicado sobre $\mathrm{F}$, sendo ambas as grandezas função de um parâmetro de configuração u. Assim, $\mathrm{f}(\mathrm{u})=\mathrm{F}(\mathrm{u}) \mathrm{C}(\mathrm{u})$ e g $=\mathrm{FC}-\mathrm{W}$, cuja diferenciação leva a

$$
\frac{\mathrm{dg}}{\mathrm{du}}=\mathrm{k}_{\mathrm{t}}=\mathrm{C} \frac{\mathrm{dF}}{\mathrm{du}}+\mathrm{F} \frac{\mathrm{dC}}{\mathrm{du}}-\frac{\mathrm{dW}}{\mathrm{du}}
$$

onde

$\frac{\mathrm{dg}}{\mathrm{du}}=\mathrm{k}_{\mathrm{t}}$ é a rigidez tangente,

$\mathrm{C} \frac{\mathrm{dN}}{\mathrm{du}}=\mathrm{k}_{\mathrm{e}}$ é a rigidez elástica,

$\mathrm{N} \frac{\mathrm{dC}}{\mathrm{du}}=\mathrm{k}_{\mathrm{g}}$ é a rigidez geométrica, e

$$
\frac{\mathrm{dW}}{\mathrm{du}}=\mathrm{k}_{\mathrm{ext}}
$$

permitindo escrever a equação (3.14) como

$$
\mathrm{k}_{\mathrm{t}}=\mathrm{k}_{\mathrm{e}}+\mathrm{k}_{\mathrm{g}}-\mathrm{k}_{\mathrm{ext}}
$$

A expressão (3.14) admite uma possível variação de $\mathrm{W}$ em função de u. Em problemas conservativos, como é o caso desta pesquisa, $\frac{\mathrm{dW}}{\mathrm{du}}=0$ e portanto $\mathrm{k}_{\mathrm{ext}}=0$. Em problemas em que as varia- 
ções geométricas são desprezíveis para efeitos da decomposição dos esforços internos, $\frac{\mathrm{dF}}{\mathrm{du}}=0$, ou seja, $\mathrm{k}_{\mathrm{g}}=0$, e o problema é geometricamente linear. Para um material de comportamento linear, $\frac{\mathrm{dF}}{\mathrm{du}}$ é constante. Sob estas três últimas hipóteses, portanto, recair-se-ia em um problema linear.

Venâncio Filho (1975) afirma que os problemas da não-linearidade geométrica conduz, como consequência, à análise da estabilidade ou cálculo da carga crítica das estruturas. Venâncio Filho (1975) realiza a construção da matriz de rigidez geométrica optando por uma formulação baseada no Princípio da Conservação da Energia via Método dos Elementos Finitos.

O Método dos Elementos Finitos (MEF) é uma contribuição original da engenharia de estruturas, que remonta aos estudos de Argyris, em 1954; Turner, Clough, Martin e Topp, em 1956; Clough, em 1960, entre outros, que utiliza o Princípio dos Trabalhos Virtuais. O MEF nada mais é que uma técnica de discretização de sistemas contínuos e aproximação numérica de suas equações diferenciais. Enraíza-se nos procedimentos do tipo Trial function utilizados nos métodos variacionais de Rayleigh (1870) e Ritz (1909) e nos de resíduos ponderados de Galerkin (1915), (BRASIL, 1995).

As estruturas reais constituem um meio contínuo de difícil equacionamento. Assim, usualmente, são utilizadas técnicas de discretização. Nelas, os deslocamentos da estrutura passam a ser descritos em função das coordenadas generalizadas adotadas. Em várias situações, um modelo adequado para representar sistemas contínuos e complexos é obtido utilizando-se um número finito de componentes simples, criando os chamados problemas discretos.

Na técnica de discretização por elementos finitos, os domínios são divididos em regiões pequenas, porém finitas, de formato simples, unidas por nós, cujos deslocamentos generalizados se tornam as incógnitas do problema. Reside aqui a diferença entre o Método dos Elementos Finitos e técnica desenvolvida neste trabalho, desenvolvida na seção 4. Enquanto que no primeiro, as funções de interpolação 
são válidas para pequenas regiões ou referências locais, no segundo, as funções de interpolação são válidas para todo o domínio.

Os deslocamentos calculados pelo Método dos Elementos Finitos multiplicam funções de interpolação de integração relativamente simples que assumem valor unitário em um dos nós, zero nos demais e no restante do domínio. Com isso, consegue-se um procedimento facilmente programável, capaz de resolver problemas de grande complexidade, sendo limitado apenas pela capacidade computacional.

Venâncio Filho (1975) aborda o problema não-linear geométrico no âmbito do Método dos Elementos Finitos da seguinte forma.

Considere-se a barra da Figura 3.6, de seção transversal constante, com momento de inércia I e comprimento $\mathrm{L}$, referida aos eixos locais $\mathrm{x}, \mathrm{y}$, com os deslocamentos nodais indicados.

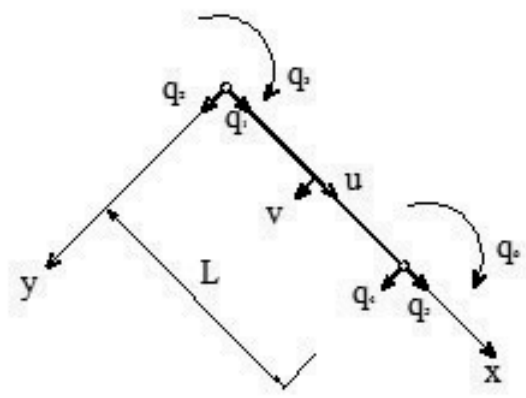

Figura 3.6 - Barra em flexão - Venâncio Filho (1975).

Para uma barra em flexão, a matriz das deformações é constituída pela curvatura do eixo da barra, à qual se associa o momento fletor. A curvatura é dada a partir do deslocamento v por

$$
-\frac{1}{\rho}=\frac{\mathrm{d}^{2} \mathrm{v}}{\mathrm{dx}^{2}}
$$

Os deslocamentos u e v podem ser expressos pelos deslocamentos nodais 


$$
\left\{\begin{array}{l}
u \\
v
\end{array}\right\}=\left[\begin{array}{cccccc}
1-\frac{x}{L} & 6\left(\frac{x}{L^{2}}-\frac{x^{2}}{L^{3}}\right) y & \left(-1+4 \frac{x}{L}-3 \frac{x^{2}}{L^{2}}\right) y & \frac{x}{L} & 6\left(-\frac{x}{L^{2}}+\frac{x^{2}}{L^{3}}\right) y & 2\left(\frac{x}{L}-3 \frac{x^{2}}{L^{2}}\right) y \\
0 & 1-3 \frac{x^{2}}{L^{2}}+2 \frac{x^{3}}{L^{3}} & x-2 \frac{x^{2}}{L}+\frac{x^{3}}{L^{2}} & 0 & 3 \frac{x^{2}}{L^{2}}-2 \frac{x^{3}}{L^{3}} & -\frac{x^{2}}{L}+\frac{x^{3}}{L^{2}}
\end{array}\right]\left\{\begin{array}{l}
\mathrm{q}_{1} \\
q_{2} \\
q_{3} \\
q_{4} \\
q_{5} \\
q_{6}
\end{array}\right\},
$$

ou seja:

$$
\{\delta\}=[\mathrm{H}]\{\mathrm{q}\},
$$

onde $\{\delta\}$ é a matriz dos deslocamentos u e v, [H] é a matriz que abriga as funções de interpolação e $\{q\}$ é a matriz coluna com os deslocamentos nodais do elemento.

A relação não-linear entre deslocamentos e deformações unitárias é, para este caso,

$$
\varepsilon=\frac{\partial \mathrm{u}_{0}}{\partial \mathrm{x}}-\frac{\partial^{2} \mathrm{v}}{\partial \mathrm{x}^{2}} \mathrm{y}+\frac{1}{2}\left(\frac{\partial \mathrm{v}}{\partial \mathrm{x}}\right)^{2}
$$

sendo $\mathrm{u}_{0} \mathrm{o}$ de valor de $\mathrm{u}$ para $\mathrm{y}=0$. A energia de deformação é, neste caso,

$$
\mathrm{U}=\frac{\mathrm{E}}{2} \int_{\mathrm{V}} \varepsilon^{2} \mathrm{dV}
$$

onde V é o volume do elemento.

Introduzindo e da equação (3.19) na equação (3.20), 


$$
\mathrm{U}=\frac{\mathrm{E}}{2} \int_{\mathrm{v}}\left[\frac{\partial \mathrm{u}_{0}}{\partial \mathrm{x}}-\frac{\partial^{2} \mathrm{v}}{\partial \mathrm{x}^{2}} \mathrm{y}+\frac{1}{2}\left(\frac{\partial \mathrm{v}}{\partial \mathrm{x}}\right)^{2}\right]^{2} \mathrm{dV}
$$

obtém-se

$$
U=\frac{E}{2} \int_{0}^{L} d x \int_{A}\left[\left(\frac{\partial u_{0}}{\partial x}\right)^{2}+\left(\frac{\partial v}{\partial x}\right)^{2} y^{2}+\frac{1}{4}\left(\frac{\partial v}{\partial x}\right)^{4}-2 \frac{\partial u_{0}}{\partial x} \frac{\partial v}{\partial x} y+\frac{\partial u_{0}}{\partial x}\left(\frac{\partial v}{\partial x}\right)^{2}-\frac{\partial^{2} v}{\partial x^{2}}\left(\frac{\partial v}{\partial x}\right)^{2} y\right],
$$

Desprezando o termo de ordem elevada $\frac{1}{4}\left(\frac{\partial \mathrm{v}}{\partial \mathrm{x}}\right)^{4}$ e tendo em vista que $\int_{\mathrm{A}} \mathrm{ydA}=0$ e $\int_{\mathrm{A}} \mathrm{y}^{2} \mathrm{dA}=\mathrm{I}$ obtém-se, efetuando a integração sobre a área na equação (3.21),

$$
\mathrm{U}=\frac{\mathrm{EA}}{2} \int_{0}^{\mathrm{L}}\left(\frac{\partial \mathrm{u}_{0}}{\partial \mathrm{x}}\right)^{2} \mathrm{dx}+\frac{\mathrm{EI}}{2} \int_{0}^{\mathrm{L}}\left(\frac{\partial^{2} \mathrm{v}}{\partial \mathrm{x}^{2}}\right)^{2} \mathrm{dx}+\frac{\mathrm{EA}}{2} \int_{0}^{\mathrm{L}} \frac{\partial \mathrm{u}_{0}}{\partial \mathrm{x}}\left(\frac{\partial \mathrm{v}}{\partial \mathrm{x}}\right)^{2} \mathrm{dx}
$$

Da equação (3.17), por outro lado, obtém-se

$$
\begin{aligned}
& \frac{\partial \mathrm{u}_{0}}{\partial \mathrm{x}}=\frac{1}{\mathrm{~L}}\left(-\mathrm{q}_{1}+\mathrm{q}_{4}\right) \\
& \frac{\partial \mathrm{v}}{\partial \mathrm{x}}=6\left(-\frac{\mathrm{x}}{\mathrm{L}^{2}}+\frac{\mathrm{x}^{2}}{\mathrm{~L}^{3}}\right) \mathrm{q}_{2}+\left(1-4 \frac{\mathrm{x}}{\mathrm{L}}+3 \frac{\mathrm{x}^{2}}{\mathrm{~L}^{2}}\right) \mathrm{q}_{3}+6\left(\frac{\mathrm{x}}{\mathrm{L}^{2}}-\frac{\mathrm{x}^{2}}{\mathrm{~L}^{3}}\right) \mathrm{q}_{5}+\left(-2 \frac{\mathrm{x}}{\mathrm{L}}+3 \frac{\mathrm{x}^{2}}{\mathrm{~L}^{2}}\right) \mathrm{q}_{6} \\
& \frac{\partial^{2} \mathrm{v}}{\partial \mathrm{x}^{2}}=\left(-\frac{6}{\mathrm{~L}^{2}}+12 \frac{\mathrm{x}}{\mathrm{L}^{3}}\right) \mathrm{q}_{2}+\left(-\frac{4}{\mathrm{~L}}+\frac{6 \mathrm{x}}{\mathrm{L}^{2}}\right) \mathrm{q}_{3}+\left(\frac{6}{\mathrm{~L}^{2}}+12 \frac{\mathrm{x}}{\mathrm{L}^{3}}\right) \mathrm{q}_{5}+\left(-\frac{2}{\mathrm{~L}}+6 \frac{\mathrm{x}}{\mathrm{L}^{2}}\right) \mathrm{q}_{6}
\end{aligned}
$$

A introdução das equações (3.23) na equação (3.22) fornece, depois de efetuada as integrações, 


$$
\begin{aligned}
& \mathrm{U}=\frac{\mathrm{EA}}{2 \mathrm{~L}}\left(\mathrm{q}_{1}^{2}-2 \mathrm{q}_{1} \mathrm{q}_{4}+\mathrm{q}_{4}^{2}\right) \\
& +\frac{2 \mathrm{EI}}{\mathrm{L}^{3}}\left(3 \mathrm{q}_{2}^{2}+\mathrm{L}^{2} \mathrm{q}_{3}^{2}+\mathrm{L}^{2} \mathrm{q}_{6}^{2}+3 \mathrm{Lq}_{2} \mathrm{q}_{3}-6 \mathrm{q}_{2} \mathrm{q}_{5}+3 \mathrm{Lq}_{2} \mathrm{q}_{5}-3 \mathrm{Lq}_{3} \mathrm{q}_{5}+\mathrm{L}^{2} \mathrm{q}_{3} \mathrm{q}_{6}-3 \mathrm{Lq}_{5} \mathrm{q}_{6}\right) \\
& +\frac{\mathrm{EA}}{\mathrm{L}^{2}}\left(\mathrm{q}_{4}-\mathrm{q}_{1}\right)\left(\begin{array}{l}
\frac{3}{5} \mathrm{q}_{2}^{2}+\frac{1}{15} \mathrm{~L}^{2} \mathrm{q}_{3}^{2}+\frac{3}{5} \mathrm{q}_{5}^{2}+\frac{1}{5} \mathrm{~L}^{2} \mathrm{q}_{6}^{2}+\frac{1}{10} \mathrm{Lq}_{2} \mathrm{q}_{3}-\frac{6}{5} \mathrm{q}_{2} \mathrm{q}_{5}+\frac{1}{10} \mathrm{Lq}_{2} \mathrm{q}_{6} \\
-\frac{1}{10} \mathrm{Lq}_{3} \mathrm{q}_{5}-\frac{1}{30} \mathrm{~L}^{2} \mathrm{q}_{3} \mathrm{q}_{6}-\frac{1}{10} \mathrm{Lq}_{5} \mathrm{q}_{6}
\end{array}\right)
\end{aligned}
$$

Considerando a força axial de compressão positiva, a força normal F é

$$
\mathrm{F}=\frac{\mathrm{AE}}{\mathrm{L}}\left(\mathrm{q}_{1}-\mathrm{q}_{4}\right)
$$

Introduzindo a Eq. (3.25) na terceira parcela da Eq. (3.24) e escrevendo-a sob forma matricial, tendo em vista que a energia de deformação de um elemento estrutural, de um modo geral, é expressa por

$$
\mathrm{U}=\frac{1}{2}\{\mathrm{q}\}^{\mathrm{T}}[\mathrm{k}]\{\mathrm{q}\}
$$

que, para o caso geométrico não-linear, torna-se

$$
\mathrm{U}=\frac{1}{2}\{\mathrm{q}\}^{\mathrm{T}}\left[\left[\mathrm{k}_{0}\right]-\left[\mathrm{k}_{\mathrm{g}}\right]\right]\{\mathrm{q}\}
$$

sendo $\{\mathrm{q}\}=\left\{\begin{array}{llllll}\mathrm{q}_{1} & \mathrm{q}_{2} & \mathrm{q}_{3} & \mathrm{q}_{4} & \mathrm{q}_{5} & \mathrm{q}_{6}\end{array}\right\},\left[\mathrm{k}_{0}\right]$ a matriz de rigidez elástica e $\left[\mathrm{k}_{\mathrm{g}}\right]$ a matriz de rigidez geométrica da barra em flexão dadas por 
$\left[\mathrm{k}_{0}\right]=\mathrm{E}\left[\begin{array}{cccccc}\frac{\mathrm{A}}{\mathrm{L}} & 0 & 0 & -\frac{\mathrm{A}}{\mathrm{L}} & 0 & 0 \\ & \frac{12 \mathrm{I}}{\mathrm{L}^{3}} & \frac{6 \mathrm{I}}{\mathrm{L}^{2}} & 0 & -\frac{12 \mathrm{I}}{\mathrm{L}^{3}} & \frac{6 \mathrm{I}}{\mathrm{L}^{2}} \\ & \frac{4 \mathrm{I}}{\mathrm{L}} & 0 & -\frac{6 \mathrm{I}}{\mathrm{L}^{2}} & \frac{2 \mathrm{I}}{\mathrm{L}} \\ & & \frac{\mathrm{A}}{\mathrm{L}} & 0 & 0 \\ \text { simétrica } & & & \frac{12 \mathrm{I}}{\mathrm{L}^{3}} & -\frac{6 \mathrm{I}}{\mathrm{L}^{2}} \\ & & & & & \frac{4 \mathrm{I}}{\mathrm{L}}\end{array}\right]$,

e

$$
\left[\mathrm{k}_{\mathrm{g}}\right]=\frac{\mathrm{F}}{\mathrm{L}}\left[\begin{array}{cccccc}
0 & 0 & 0 & 0 & 0 & 0 \\
& \frac{6}{5} & \frac{\mathrm{L}}{10} & 0 & -\frac{6}{5} & \frac{\mathrm{L}}{10} \\
& & \frac{2 \mathrm{~L}^{2}}{15} & 0 & -\frac{\mathrm{L}}{10} & -\frac{\mathrm{L}^{2}}{30} \\
& & & 0 & 0 & 0 \\
\text { simétrica } & & & \frac{6}{5} & -\frac{\mathrm{L}}{10} \\
& & & & & \frac{2 \mathrm{~L}^{2}}{15}
\end{array}\right],
$$

A matriz da Equação (3.29) denota a dependência da rigidez geométrica com o esforço normal que age em seus extremos e com o comprimento do elemento.

Para uma primeira aproximação dos efeitos da não-linearidade, os postes de telefonia celular podem perfeitamente ser modelados por um sistema composto por uma barra engastada na base. 
Considere-se uma barra livre em uma extremidade e engastada na outra, com os deslocamentos nodais indicados na Figura 3.7.

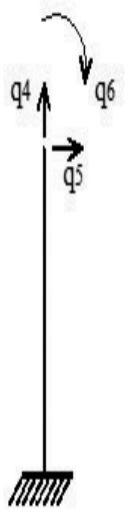

Figura 3.7 - Graus de liberdade de um elemento de barra.

Para se analisar o comportamento dinâmico sob não-linearidade geométrica desse sistema, nas proximidades da configuração indeformada, ou original, a matriz de rigidez, com base em (3.28) e (3.29) é

$$
[\mathrm{K}]=\left[\begin{array}{ccc}
\frac{\mathrm{EA}}{\mathrm{L}} & 0 & 0 \\
\text { simétrica } & \frac{12 \mathrm{EI}}{\mathrm{L}^{3}} & -\frac{6 \mathrm{EI}}{\mathrm{L}^{2}} \\
& & \frac{4 \mathrm{EI}}{\mathrm{L}}
\end{array}\right]-\frac{\mathrm{F}}{\mathrm{L}}\left[\begin{array}{ccc}
0 & 0 & 0 \\
\text { simétrica } & \frac{6}{5} & -\frac{\mathrm{L}}{10} \\
& \frac{2 \mathrm{~L}^{2}}{15}
\end{array}\right]
$$

onde E é o módulo de elasticidade do material, A é a área da seção transversal, L é o comprimento da barra, I é o momento de inércia da seção em relação ao eixo perpendicular ao plano que contém a figura e F é o esforço normal de compressão tomado como positivo.

É possível realizar uma simplificação da matriz de rigidez, através da condensação estática, onde a rotação é expressa em termos da translação na direção do movimento, e considerar exclusivamente o grau de liberdade livre horizontal, o que reduz o sistema a um grau de liberdade e a matriz a um único termo: 


$$
\mathrm{K}=\frac{3 \mathrm{EI}}{\mathrm{L}^{3}}-1,125 \frac{\mathrm{F}}{\mathrm{L}}
$$

As matrizes elástica e geométrica, apresentadas em (3.28) e (3.29), foram desenvolvidas no sistema local do elemento. Quando se trata de estruturas com sistema discreto de coordenadas generalizadas é preciso referi-las ao sistema de referência global obedecendo à correlação existente entre os deslocamentos nos dois sistemas. As técnicas desse procedimento podem ser encontradas em Cook (2002) e Bathe (1996).

Quando excitados, os sistemas estruturais estarão sujeitos a forças conservativas e forças dissipativas em maior ou menor grau dependendo de suas características geométricas, materiais e do meio onde se encontra inserido. Desprezando-se os atritos, intrínseco do material e do meio, e uma vez cessada a excitação, os únicos movimentos possíveis, nessa situação, devem-se às condições iniciais de deslocamento e de velocidade.

Simiu; Scalan (1996) afirmam que um sistema estrutural com baixo amortecimento, quando excitados pelo vento, irão vibrar em ressonância com as formas definidas por suas frequências naturais. As formas (modos) e as frequências naturais de vibração são propriedades da estrutura, independem da excitação, estão relacionados à maneira de como se dá a distribuição de massa do sistema e à rigidez da estrutura.

As equações de movimento livre não amortecido de um sistema com vários graus liberdade, referidas ao sistema discreto de coordenadas generalizadas, podem ser escritas na forma:

$$
[\mathrm{M}]\{\ddot{\mathrm{x}}\}+[\mathrm{K}]\{\mathrm{x}\}=0
$$

onde 
$[\mathrm{M}]$ é a matriz de massa;

$\{\ddot{x}\}$ é o vetor aceleração;

[K] é a matriz de rigidez, compreendida por $\mathrm{K}=\mathrm{K}_{0}+\mathrm{K}_{\mathrm{g}}$ que introduz a não-linearidade geométrica na equação do movimento $\left(\mathrm{K}_{0}\right.$ é matriz de rigidez à elástica e $\mathrm{K}_{\mathrm{g}}$ é a matriz de rigidez geométrica);

$\{\mathrm{x}\}$ é o vetor deslocamento;

A Eq.(3.32) é uma equação diferencial homogênea, cujas soluções são formas $\{\Phi\}$, que representam os modos de vibração em que todas as coordenadas do sistema variam na mesma frequência e harmonicamente no tempo. Escrevem-se, portanto, os deslocamentos nodais como

$$
\{\mathrm{x}\}=\{\Phi\} \cos (\omega \mathrm{t}-\theta)
$$

A Eq. (3.33) representa a vibração do sistema segundo um modo normal de vibração, o correspondente à frequência $\omega$. Derivando essa solução duas vezes no tempo, substituindo na equação (3.33) e cancelando a função harmônica, recai-se no sistema de equações algébricas homogêneas

$$
\left[[\mathrm{K}]-\omega^{2}[\mathrm{M}]\right]\{\Phi\}=0
$$

Para que sejam possíveis soluções não-triviais, o determinante da matriz deve ser nulo

$$
\operatorname{det}\left[[\mathrm{K}]-\omega^{2}[\mathrm{M}]\right]=0
$$


resultando numa equação polinomial de grau $n$ na variável $\omega^{2}$, conhecida como equação de frequência. As $n$ soluções $\omega_{i}$, neste caso, são reais e positivas e são as frequências naturais do sistema. Usualmente, denota-se por $\omega_{1}$ a menor delas e, pela ordem, até a maior $\omega_{n}$. Assim, podem-se determinar os $n$ modos de vibração e colecioná-los numa matriz modal $n \times n$, cujas colunas são os $n$ modos de vibração livres não amortecidos, normalizados (Brasil, 2004). Cada par de autovalores/autovetores é conhecido como uma frequência e um modo de vibração do sistema. As frequências cíclicas em Hz serão

$$
\mathrm{f}_{\mathrm{i}}=\frac{\omega_{\mathrm{i}}}{2 \pi}
$$

cujos inversos são os períodos de vibração livres em segundos.

Para considerar todos os valores e vetores característicos em número igual ao de deslocamentos nodais do sistema, Venâncio Filho (1975) sugere escrever a Eq. (3.34) como

$$
[\Phi]\left[\omega^{2}\right]=[\mathrm{K}][\mathrm{M}]^{-1}[\Phi]
$$

onde $\left[\omega^{2}\right]$ é a matriz diagonal de ordem $n$ constituída pelas frequências naturais ao quadrado e [Ф] a matriz nxn cujas colunas são os modos normais de vibração. A matriz $[\mathrm{K}][\mathrm{M}]^{-1}$ é chamada de matriz dinâmica, também mencionada por Blessmann (2005).

No caso de distribuição discreta de massa ("lumped mass"), a matriz de massa do sistema estrutural é simplesmente uma matriz diagonal constituída pelas massas e momentos de inércia concentrados relativos aos deslocamentos nodais. Os problemas discretos constituem a base das análises realizadas para a determinação da resposta da estrutura à turbulência atmosférica.

Na determinação da resposta dinâmica das estruturas sob excitação de vento, os modos naturais de vibração desempenham papel 
fundamental. A solução desenvolvida para o cálculo da força estática equivalente à ação dinâmica do vento baseia-se em um tratamento estatístico sobre a equação do movimento escrita com os modos normais de vibração do sistema discretizado, para as quais tanto a matriz de massa quanto as de rigidez são diagonais.

Como explicitado em (3.32), fica claro que a diferença entre a determinação da frequência de sistemas lineares e não-lineares do ponto de vista geométrico se fundamenta na construção da matriz de rigidez. Enquanto que no primeiro caso apenas os efeitos elásticos da matriz de rigidez são levados em conta, no segundo a matriz de rigidez total depende da parcela geométrica, que é função do esforço normal atuante.

Wilson e Habibullah (1987) afirmam que a utilização da matriz de rigidez na dinâmica estrutural é uma técnica viável para o cálculo dos efeitos de segunda ordem, pois esse efeito é linearizado e a solução do problema é obtida diretamente, de forma exata, sem iterações. É válida para situações onde a força vertical devido ao peso próprio e sobrecargas permanecem constantes durante o movimento da estrutura e para aquelas situações, cujos deslocamentos laterais sofridos são pequenos quando comparados às suas dimensões.

Nesse contexto, somente o peso próprio da estrutura e as sobrecargas verticais necessitam ser incluídas na parcela negativa da rigidez geométrica. Esse método se aplica tanto a cálculos estáticos quanto dinâmicos, consistindo em um processo que pode ser facilmente programável no ambiente do Método dos Elementos Finitos, exigindo reduzido esforço computacional. Medeiros e França (1989) utilizaram essa facilidade de programação e aliaram o Método dos Elementos Finitos à análise não-linear simplificada na solução de diversos problemas da engenharia, compararam os resultados obtidos a métodos analíticos e outros métodos de análise não-lineares, concluindo pela eficiência do método.

Além do mais, como expõe Rutenberg (1982), as cargas gravitacionais sobre as colunas dos edifícios são relativamente baixas quando comparadas à carga crítica de Euler, assim como os efeitos adicionais de segunda ordem, permitindo que se aplique uma solução aproximada via matriz de rigidez geométrica, linearizando o problema. 
As estruturas civis são corpos sujeitos a esforços aos quais devem resistir para que a sua forma se mantenha razoavelmente próxima das configurações desejadas, durante os movimentos induzidos, ou seja, os movimentos de uma estrutura civil devem ser pequenos em torno de uma configuração projetada, portanto, a análise dinâmica sob não-linearidade geométrica realizada por meio da matriz de rigidez é perfeitamente cabível. 